EUROPHYSICS LETTERS

1 January 1996

Europhys. Lett., 23 (1), pp. 1-5 (1993)

\title{
Phase separation in systems with absorbing states.
}

\author{
Miguel A. Muñoz ${ }^{1}$, U. Marini Bettolo Marconi ${ }^{1,2}$ and R. Cafiero ${ }^{1,3}$ \\ 1 I.N.F.M. Sezione di Roma 1, and Dipartimento di Fisica, Universitá di Roma "La \\ Sapienza", Piazzale A. Moro 2, I-00185 Roma, Italy \\ 2 I.N.F.M. Sezione di Camerino and Dipartimento di Matematica e Fisica, Università \\ di Camerino, Via Madonna delle Carceri, I-62032, Camerino, Italy \\ 3 Max-Planck Institut für Physik komplexer Systeme, Nöthnitzer Strasse 38, D-01187 \\ Dresden, Germany \\ (received ; accepted ) \\ PACS. 64.60.-i - General studies of phase transitions. \\ PACS. 64.60.Cn- Order disorder transformations.
}

\begin{abstract}
We study the problem of phase separation in systems with a positive definite order parameter, and in particular, in systems with absorbing states. Owing to the presence of a single minimum in the free energy driving the relaxation kinetics, there are some basic properties differing from standard phase separation. We study analytically and numerically this class of systems; in particular we determine the phase diagram, the growth laws in one and two dimensions and the presence of scale invariance. Some applications are also discussed.
\end{abstract}

The presence of conservation laws plays a key role in determining the phenomenology and critical behavior of phase transitions both in equilibrium and nonequilibrium systems. For instance, the dynamics of models for magnetism change dramatically depending on whether the order parameter is a conserved quantity or not [1]. In the conserving case, models may exhibit phenomena such as spinodal decomposition (SD hereafter) and droplet growth via condensation-evaporation [2, 3, 旬, which are absent in models with non-conserving dynamics. The problem of SD that appears, for instance in binary alloys, polymers and spin systems with conserved magnetization, have attracted a lot of interest in the last decades [2, 3, 1, and despite the efforts devoted to understand their phenomenology, some of their aspects remain unclear. The key issue is that of understanding the dynamics of a system quenched from a state in the homogeneous phase into a broken-symmetry phase. Depending on the value of the conserved order parameter, $M$, different types of morphologies can show up. The archetypal field theory representing this class of systems is the model B (or Cahn-Hilliard equation), defined by the following Langevin equation

$$
\partial_{t} \phi(x, t)=\nabla^{2} \frac{\delta F[\phi(x, t)]}{\delta \phi(x, t)}+\nabla \cdot \eta(x, t)
$$

Typeset using EURO-LATEX 
where $F[\phi(x, t)]$ is a free energy functional given by

$$
F[\phi]=\int d x\left[-\frac{a}{2} \phi^{2}(x)+\frac{b}{4} \phi^{4}(x)+\frac{1}{2}(\nabla \phi(x))^{2}\right]
$$

with $a$ and $b$ positive constants, and $\eta(x, t)$ is a d-dimensional noise vector whose components obey

$$
\left\langle\eta_{i}(x, t)\right\rangle=0, \quad\left\langle\eta_{i}(x, t) \eta_{j}\left(x^{\prime}, t^{\prime}\right)\right\rangle=D \delta^{(d)}\left(x-x^{\prime}\right) \delta\left(t-t^{\prime}\right) \delta_{i, j} .
$$

A brief discussion of its phenomenology is as follows: an homogeneous initial condition with averaged field density $\phi_{0}$, located in the unstable zone of the free energy, experiences a linear instability which favors the growing of Fourier modes with $k \neq 0$ (waves), and the initially homogeneous phase roughens, in such a way that locally the system tries to relax to one of the two minima of the free energy, conserving the total order parameter. Let us point out that the presence of two different minima in Eq. (2) is a key ingredient for phase separation. After an initial stage the wave amplitude grows, until it saturates, i.e. the maxima and minima reach the maximum and minimum of the free energy potential, and the symmetry with respect to the initial value of the field is lost [5]. Afterwards the clusters of the minority phase coalesce via diffusive annihilation of interfaces. For metastable initial conditions the system is locally stable, but fluctuations can take the local order parameter to one of the minima, in this way droplets are formed, and they grow via nucleation.

In a seminal work Lifshitz and Slyozov [6], and independently Wagner [7], derived some exact results for very diluted quenches, i.e. when the volume fraction of one phase is much larger than the other one. For example, the averaged gyration radius of clusters in the minority phase, $R$, grows with time as $R \approx t^{1 / 3}$, while it grows like $t^{1 / 2}$ in the non-conserved case. These results have been shown to be independent of the spatial dimension $d$ [8] and confirmed in numerical studies [9, 10]. Although the Lifshitz-Slyozov-Wagner theory (LSW) is only valid for very dilute systems, it has been argued that a similar law $t^{1 / 3}$ is valid also for generic volume fractions [11]. An intermediate regime has also been identified in some cases [12]; it is characterized by the presence of soft domain walls and a growth law $t^{1 / 4}$; after this regime the domain walls harden (become sharper) and the real asymptotic behavior $t^{1 / 3}$ sets in. In other cases in which bulk diffusion is stopped the assymptotic domain growth law is characterized by an exponent $1 / 4$ 13.

Another important result is the identification of a single relevant length scale in the late stages of phase separation. It is given by the averaged droplet size. This makes it possible to describe the late stages of growth in terms of scaling functions [2, 10, 14, 15], and makes the problem suitable to be studied using renormalization group techniques 16 , 1 . It is accepted that in the scaling regime stochastic effects are irrelevant and therefore the same behavior is obtained in purely deterministic systems with the same symmetries and conservation laws.

In this paper we face the generalization of the previous ideas to a vast class of nonequilibrium systems, namely, the class of systems with absorbing states. It is well established that all the systems exhibiting a continuous transition into a unique absorbing state, without any other extra symmetry or conservation law, belong to the same universality class, namely, that of directed percolation 17, 18. Among other models in that broad class are the following: directed percolation, the contact process, catalytic reactions on surfaces, branching annihilating random walks, damage spreading and self organized criticality (see [17, 18] and references therein). The Reggeon field theory (RFT) is the minimal continuous theory in this vast universality class [18. In its conserved version it is defined by a Langevin equation analogous to Eq. (11) where 
now $F[\phi(x, t)]$ is a different free energy functional given by

$$
F[\phi]=\int d x\left[-\frac{a}{2} \phi^{2}(x)+\frac{b}{3} \phi^{3}(x)+\frac{1}{2}(\nabla \phi(x))^{2}\right],
$$

and

$$
\left\langle\eta_{i}(x, t)\right\rangle=0, \quad\left\langle\eta_{i}(x, t) \eta_{j}\left(x^{\prime}, t^{\prime}\right)\right\rangle=D \phi(x, t) \delta^{(d)}\left(x-x^{\prime}\right) \delta\left(t-t^{\prime}\right) \delta_{i, j} .
$$

The main properties of this equation in contrast to those of model B are: (i) all the dynamics ceases for $\phi(x)=0$, as corresponds to a system with an absorbing state; (ii) the RFT describes the dynamics of a density like, positive definite, order parameter, and (iii) no up-down symmetry is present in this type of systems and therefore the first nonlinearity in the free energy is a cubic term instead of a quartic one as in model B (observe, however, that none of the forthcoming results depend on the degree of the non-linearity).

Owing to these last two differences it is clear that the essential mechanisms present in standard phase separation cannot straightforwardly be translated to the conserved RFT: The deterministic free energy $F$ exhibits only one minimum (at 0 if $a \leq 0$, and at $\phi=a / b$ if $a>0$ ), and the process of phase separation, if present, does not correspond to a local relaxation of the density to the two minima of the local free energy as in model B.

Before proceeding further, let us present a simple toy model in economics that shares the properties of our previously described system (as described by Eq. (1), (4) and (5). This toy model can be view as a discretization of our proposed equations, and maybe be useful to develop an intuition of the forthcoming conclusions and results. The model is defined on a 2-dimensional discrete lattice, each site representing an individual. A field variable describes the wealth of each individual at each time. The wealth is updated at each time step following three different mechanisms (1) the wealth is driven by an external agent (a fair government) that tries to keep all the individual equally rich and corresponds to the potential in our model; (2) the wealth is redistributed periodically among nearest neighbors ( this corresponds to the Laplacian in (仼) and describes fair citizens), and (3) in addition there are random fluctuations. The dynamics is local (wealth flows only through nearest neighbors) and conserves the total wealth. The problem is whether the individuals will remain equally rich on average or not with these utopian rules. As we will show in what follows the answer depends on the total wealth of the society.

Coming back to the general problem, a straightforward analysis of local stability of our continuous model shows that the homogeneous state is unstable with respect to fluctuations whenever $\phi<a / 2 b$. The problem arises of what is the kinetics in this case; if the system segregates in different phases what do they correspond to?

By Fourier transforming the Langevin equation, it is easy to verify that the fastest growing mode is $k_{m}=\sqrt{\left(a-2 b \phi_{0}\right) / 2}$, and therefore the fastest growing length, given by $\lambda=2 \pi / k_{m}$ is $\lambda=2 \pi / \sqrt{\left(a-2 b \phi_{0}\right) / 2}$. The system size has to be larger than $\lambda$ for the instability to be observed; for finite systems of size $L$, the effective instability point is shifted from $\phi_{0}=a / 2 b$ to a smaller value $\phi_{0} \approx\left(a-\frac{2(2 \pi)^{2}}{L}\right) / 2 b$, determined by the condition $k_{m}=L$.

For parameter values in the locally unstable regime initial homogeneous conditions break down, and a wavy solution dominated by the wave vector $k_{m}$ sets in. This wavy solution cannot grow in amplitude indefinitely due to the physical constraint at $\phi=0$; and, in contrast to what happens in standard SD the maxima and minima of the wavy solution do not collapse to the two minima of the deterministic potential (which in our case is monostable). Therefore, if the system is unstable the question arises of what is the true stable solution. In order to answer the same question for the model $\mathrm{B}$, one can solve the equation $\delta F[\phi] / \delta \phi=0$ with $F[\phi]$ given by Eq. (2). A well known solution of the previous equation is $\phi(x)=\sqrt{a / b} \tanh (\sqrt{a} x /(2 b))$. 
This is a topological solution connecting the two minima of $F$. In our case such a topological solution cannot exist due to the presence of only one minimum in $F$. This, however, does not mean that the full stationary equation, i.e. $\nabla^{2}(\delta F[\phi] / \delta \phi)=0$ does not admit a topological solution, even though in this case it is a much less intuitive one. Let us look for such a solution, and let us do it first in $d=1$ (the generalization to higher dimensions being straightforward). The general solution of the Laplace equation, $\nabla^{2} f(x)=0$ in $d=1$ is $f(x)=h+g x$ where $h$ and $g$ are undetermined constants; therefore a solution of the stationary problem is also a solution of $-\frac{\delta F}{\delta \phi}=h+g x$ (where we have just replaced $f$ by $-\frac{\delta F}{\delta \phi}$ ). Imposing periodic boundary conditions, we can fix $g=0$, while $h$ is specified by the initial condition, $h V=-\left.\int d x \frac{\delta F}{\delta \phi}\right|_{\phi=\phi_{0}}$ where $V$ is the system volume (which transforms into $h=a \phi_{0}-b \phi_{0}^{2}$ with $\phi_{0}=\phi(t=0)$ for homogeneous initial conditions). Observe that the previous equation, $-\frac{\delta F}{\delta \phi}=h$, can be rewritten as $\frac{\delta G}{\delta \phi}=0$, with $G[\phi]=F[\phi]+h \phi$, and therefore the effect of the initial condition is to originate a constant effective field, $h$, coupled linearly to the field variable. We can now look for solutions of this modified equation, which may have two minima. In fact, the morphology of the new deterministic potential (in terms of $G$ ) is as follows:

(i) For $\phi_{0}>a / b, \mathrm{~h}$ is negative, and the effective potential has only a minimum (there is another, nonphysical extremum of the effective potential located at some negative value of $\phi$ ).

(ii) At $a / b \geq \phi_{0} \geq a / 2 b$ the effective potential develops a maximum at $\phi_{M}=a / b-\phi_{0}$, and the minimum is at $\phi_{m}=\phi_{0}$. Between $\phi=0$ and $\phi_{M}$ the potential is a monotonously increasing function of $\phi$, therefore at $\phi=0$ the potential exhibits a relative minimum even though its derivative is not zero. As the value of $\phi_{0}$ decreases the maximum and the minimum approach each other.

(iii) For $a / 2 b \geq \phi_{0} \geq 0$, the two extrema (that collapse into a saddle point at $\phi_{0}=a / 2 b$ ) start separating again as $\phi_{0}$ is reduced. The maximum, located now at $\phi_{M}=\phi_{0}$ returns to 0 at $\phi_{0}=0$, while the minimum, $\phi_{m}=a / b-\phi_{0}$, increases as $\phi_{0}$ is decreased.

Observe that in the interval $[0, a / b]$ the effective potential has the same form at $a / 2 b+\Delta$ and at $a / 2 b-\Delta$. Note also that in that interval the potential has two minima, one with zero derivative, and another at the origin with, in general, non-vanishing derivative, which is due to the presence of an absorbing barrier at $\phi=0$. It is also possible to decide the fraction of total density located at each minima; supposing in first approximation that the potential is locally minimized at all the points simultaneously, and calling $p$ the fraction at $\phi=0$ and $1-p$ the complementary probability in such a way that $\phi_{0}=0 p+(1-p) \phi_{m}$, we obtain $p=\left(a-2 b \phi_{0}\right) /\left(a-b \phi_{0}\right)$. In particular the mixture is symmetric at $\phi_{0}=a /(3 b)$.

We have performed some numerical simulations to further study the proposed model. For that we consider an initial condition with average field density $\phi_{0}$ and small fluctuations around that value. In figure 1 we show a long time typical density profile in $d=1(L=100)$ for two different initial densities in the unstable regime: $\phi_{0}=0.20$ and $\phi_{0}=0.26$. The two phases are clearly asymmetric; the phase at $\phi=0$ is free of fluctuations. At $\phi_{0}=0.26$ ( i.e. slightly below the critical point of instability, $a / 2 b=0.3$ for the chosen parameter values) the fraction of sites in the nonvanishing region is larger than in the other case, but in any case, the walls are quite soft.

We have verified the presence of only one characteristic length is the scaling regime. For that we have computed the structure function at different times, and rescaled it using $R(t)$; a good collapse of the curves is observed (see Fig.2).

For the time evolution of the averaged cluster size we get, in $d=1: R(t) \sim t^{(\alpha \pm 0.02)}$, with $\alpha=0.30,0.28$ and 0.26 , for initial concentrations $\phi_{0}=0.05,0.1$ and 0.15 respectively (Fig.3).

Deciding whether the assymptotic behavior is governed by an exponent $1 / 3$ or $1 / 4$ is a difficult task from both theory and numerics. From the theoretical point of view it has been argued that when the bulk dynamics is suppressed an exponent $1 / 4$ can be obtained [13], while 


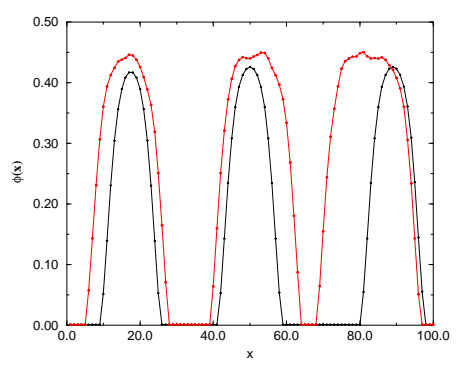

Fig. 1. - Density profile in the one-dimensional case for $\phi_{0}=0.20$ (lower curve) and $\phi_{0}=0.26$ (upper curve) respectively at $t \approx 10^{6}$. The parameter values are taken to be: $a=0.6, b=1$, and $D=0.01$.

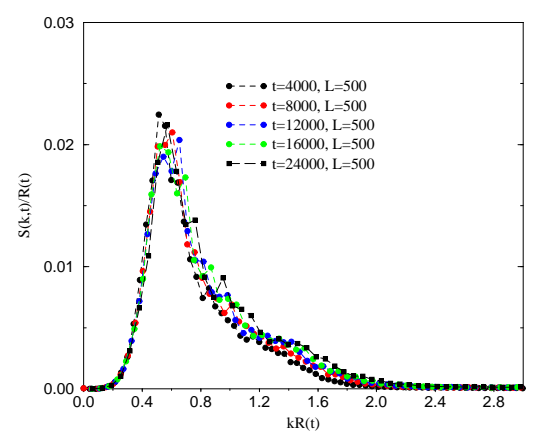

Fig. 2. - Rescaled structure function in $d=1$ for times from $t=4000$ to $t=24000$.

time dependent exponents, interpolating between $1 / 3$ and $1 / 4$ (reflecting crossover effects) are obtained for concentration dependent diffusion coefficient 13 . Observe that in our model the dynamics ceases in the interior of absorbing clusters but not in the other (active) phase. More systematic simulations and further theoretical analysis aiming to sort out this point will be presented elsewhere.

In $d=2$ a similar qualitative behavior is obtained. In Fig.4 we show the evolution of the field variable as a function of time for an initial density $\phi_{0}=0.05$. Observe that first absorbing (black) regions are created in a sea of active sites (white) (at time $t=0$ the whole lattice would be white). Afterwards these islands percolate through the system and the active regions become isolated.

All the measured effects in both $d=1$ and $d=2$, are essentially deterministic, and correspond therefore to the $T=0$ fixed point of the conserved RFT. Therefore, the main ingredient characterizing this phase separation in systems with an absorbing state, is the physical constraint at $\phi=0$, and not the noise itself.

Our system may exhibit a phase transition from the ordered behavior to a disordered phase as the noise amplitude is increased. Due to the presence of a noise of a different nature we expect this transition to be in a universality class other than that of model $\mathrm{B}$. This issue will be analyzed in a forthcoming paper.

Summing up, we have shown that deterministically monostable systems, and, in particular, 
EUROPHYSICS LETTERS

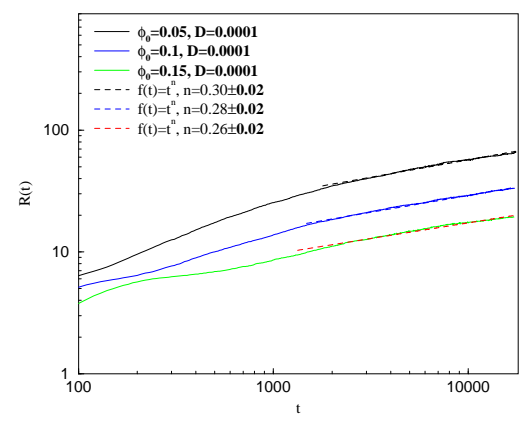

Fig. 3. - Log-log plot of the averaged mean cluster radius as a function of time for $d=1$, and different initial densities.

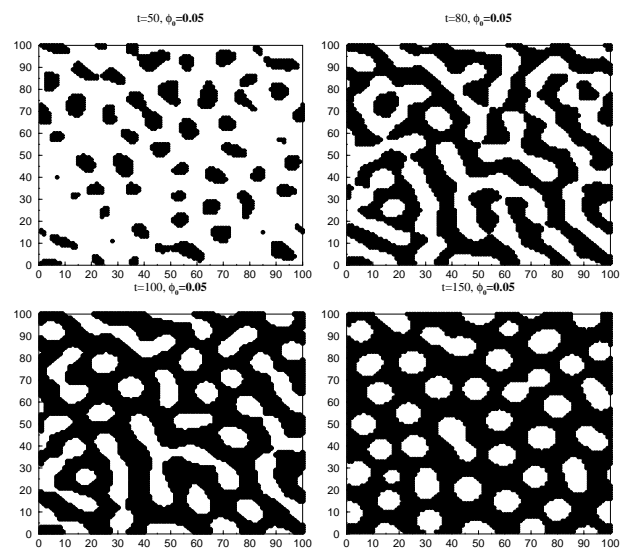

Fig. 4. - Time evolution of the density in a $100 \times 100$ lattice for an initial average concentration $\phi_{0}=0.05$ (with small fluctuations around that mean value). Black points represent sites in the absorbing state, while active regions (i.e. above a certain tiny threshold) are represented in black. First the initial condition in unstable and absorbing regions are created $(t=50)$. Then these regions coalesce $(t=80)$ and percolate through the system, isolating regions of active sites $(t=100)$. The size of active regions shrinks down for late times $(t=150)$.

systems with absorbing barriers can exhibit the phenomenon of phase separation, in a form that shows many common features with the standard picture of phase separation, but has also some essential differences. These come mainly from the fact that the two minima of the effective potential are intrinsically of different nature in our model.

Among other many possible applications of the general phenomena we have just described let us cite the following: phase separations of charged particles in a conductor (observe that these situation has an absorbing phase: zones with no charge are fluctuation free), or diffusion of particles in an absorbing substrate. We expect the general scenario studied here to describe some realistic model similar to the ones cited previously.

Finally, coming back to our toy model in economics, the wealth remains homogeneously distributed among individuals when the total wealth in the society is larger than a critical value, (determined by the government tax policy), but when it is smaller than the critical 
threshold the society becomes separated in two groups: rich and poor, which separate from each other.

It is a pleasure to acknowledge useful discussions with J. M. Sancho J. Marro, P. Garrido, L. Pietronero, and R. Toral. This work has been partially supported by the European Union through a grant to M.A.M. (contract ERBFMBICT960925).

\section{REFERENCES}

[1] P. C. Hohenberg and B. I. Halperin, Rev. Mod. Phys., 49 (1977) 435 and references therein.

[2] J. D. Gunton, M. San Miguel, and P. S. Sahni, in Phase Transitions and Critical Phenomena Vol. 8, edited by C. Domb and J. L. Lebowitz p. 267,( New York: Academic Press) 1983

[3] J. S. Langer,, Solids Far From Equilibrium, edited by C. Godreche, (Cambridge University Press), p.297. p. 2971992

[4] A. J. Bray, Adv. in Phys., 43 (1994) 357

[5] S. Joly, A. Raquois, F. Paris, B. Hamdoun, L. Auvary, and D. Ausserre, Phys. Rev. Lett., 77 (1996) 4394

[6] I. M. Lifshitz And V. V. Slyozov, J. Phys. Chem. Solids, 19 (1961) 35

[7] C. Wagner, Z. Electrochem., 65 (1961) 581

[8] K. Binder, Phys. Rev. B, 25 (1977) 4425

[9] T. M. Rogers and R. C. Desai, Phys. Rev. B, 39 (1989) 11956; T. M. Rogers, K. R. Elder, and R. C. Desai, Phys. Rev. B, 37 (1988) 9638

[10] A. Chakrabarti, R. Toral, and J. Gunton, Phys. Rev. E, 47 (1993) 3025

[11] D. A. Huse, Phys. Rev. B, 34 (1986) 7845; A. J. Bray, Phys. Rev. Lett., 62 (1989) 2841

[12] Y. Oono and S. Puri, Phys. Rev. Lett., 58 (1987) 836

[13] A. M. Lacasta, A. Hernández-Machado and J. M. Sancho, Phys. Rev. B, 45 (1992) 5276

[14] J. Marro, J. L. Lebowitz, and M. H. Kalos, Phys. Rev. Lett., 43 (1979) 282

[15] H. Furukawa, Adv. in Phys., 34 (1985) 703

[16] G. F. Mazenko, O. T. Valls, and F. C. Zhang, Phys. Rev. B, 31 (1985) 4453; ibid 32 (1985) 5807

[17] J. Marro And R. Dickman, Nonequilibrium Phase Transitions and Critical Phenomena,(Cambridge University Press) (1996)

[18] See G. Grinstein And M. A. Muñoz, The Statistical Mechanics of Absorbing States, on Third Granada seminar on Computational Physics, Ed. P. Garrido and J. Marro. Lecture Notes in Physics, 49, p.223, (Springer, Berlin) 1997, and references therein. 